\title{
Pillar-type acoustic metasurface
}

Jin, Yabin; Bonello, Bernard; Moiseyenko, Rayisa; Pennec, Yan; Boyko, Olga; Djafari-Rouhani, Bahram

\section{Published in:}

Physical Review B

Link to article, DOI:

10.1103/PhysRevB.96.104311

Publication date:

2017

Document Version

Publisher's PDF, also known as Version of record

Link back to DTU Orbit

Citation (APA):

Jin, Y., Bonello, B., Moiseyenko, R., Pennec, Y., Boyko, O., \& Djafari-Rouhani, B. (2017). Pillar-type acoustic metasurface. Physical Review B, 96(10), [104311 ]. https://doi.org/10.1103/PhysRevB.96.104311

\section{General rights}

Copyright and moral rights for the publications made accessible in the public portal are retained by the authors and/or other copyright owners and it is a condition of accessing publications that users recognise and abide by the legal requirements associated with these rights.

- Users may download and print one copy of any publication from the public portal for the purpose of private study or research.

- You may not further distribute the material or use it for any profit-making activity or commercial gain

- You may freely distribute the URL identifying the publication in the public portal

If you believe that this document breaches copyright please contact us providing details, and we will remove access to the work immediately and investigate your claim. 


\title{
Pillar-type acoustic metasurface
}

\author{
Yabin Jin, ${ }^{1,2}$ Bernard Bonello, ${ }^{3, *}$ Rayisa P. Moiseyenko, ${ }^{4}$ Yan Pennec, ${ }^{1}$ Olga Boyko, ${ }^{3}$ and Bahram Djafari-Rouhani ${ }^{1}$ \\ ${ }^{1}$ Institut d'Electronique, de Micro-électronique et de Nanotechnologie (IEMN-UMR CNRS 8520), Université de Lille1, \\ UFR de Physique, Cité Scientifique, 59652 Villeneuve d'Ascq Cedex, France \\ ${ }^{2}$ Institut de Mécanique et d'Ingénierie (I2M-APY, UMR CNRS 5295), Université de Bordeaux, 33405 Talence, France \\ ${ }^{3}$ UPMC Université Paris 6, UMR CNRS 7588, INSP, 75005 Paris, France \\ ${ }^{4}$ Department of Applied Mathematics and Computer Science, Technical University of Denmark, Richard Petersens Plads, \\ Building 324, 2800 Kgs. Lyngby, Denmark
}

(Received 28 April 2017; revised manuscript received 14 September 2017; published 28 September 2017)

\begin{abstract}
We theoretically investigate acoustic metasurfaces consisting of either a single pillar or a line of identical pillars on a thin plate, and we report on the dependence on the geometrical parameters of both the monopolar compressional and dipolar bending modes. We show that for specific dimensions of the resonators, bending and compressional modes may be simultaneously excited. We study their interaction with an antisymmetric Lamb wave, whether or not they occur at the same frequency, with particular consideration for the amplitude and phase of waves emitted by the pillars at resonance. Especially, the analysis of both the amplitude and the phase of the wave at the common resonant frequency downstream from a line of pillars demonstrates that the reemitted waves allow for the transmission with phase shift of $\pi$.
\end{abstract}

DOI: 10.1103/PhysRevB.96.104311

\section{INTRODUCTION}

Locally resonant sonic materials are artificially structured composites designed to exhibit negative effective mass density and/or elastic constants at some frequencies [1]. They are based on the insertion into a background (3D) or on a free surface (2D) of local resonators having lateral sizes much smaller than the wavelength of the elastic wave so that homogenization theories apply. Unusual responses are then observed: the material expands upon compression when the compressibility gets negative and moves to the left when pushed toward the right if the mass density is negative. Although the periodicity is not a requirement, these resonators are generally regularly arranged and therefore Bragg scattering may occur when the acoustic wavelength is of the order of the spacing between the inclusions. Besides this expected property, flat bands related to the normal modes of the resonators occur in the band structure at much lower frequencies as compared to the Bragg band gap. This property was first recognized in the seminal work of Liu et al. [2] and further exploited by Li and Chan [3], who demonstrated the double negativity in a phononic crystal made of rubber spheres arranged in a fcc lattice in water. In both systems, the negative compressibility resulted from a monopolar resonance of the rubber spheres, whereas a dipolar resonance yielded to the negative mass density. Very recently, negative density in a liquid foam [4] and negative acoustic refractive index in Mie resonators made of random suspension of soft silicone rubber microbeads $[5,6]$ have been demonstrated. Both effective mass density and effective bulk modulus become negative when the resonators are in simultaneous dipolar and monopolar motions, out-of-phase with respect to the waves propagating in the background. If, moreover, the geometrical parameters of the crystal (diameter of the spheres, filling ratio ... ) are chosen in such a way that the mass density and the compressibility are

*Corresponding author: bernard.bonello@insp.jussieu.fr both negative in the same frequency band, the elastic waves become propagative and the transmission coefficient through the entire structure goes to unity. However, all these unique properties are achievable only because the speed of sound in the soft rubber or in the bubbly liquid is of a few tens of $\mathrm{m} \mathrm{s}^{-1}$, lower by orders of magnitude than the speed of sound in the epoxy matrix [2,7] or in the water background [3]. Actually, the smaller the contrast in sound speeds, the smaller the wavelength in the embedding matrix at resonance and the phononic crystal can no longer be viewed as an effective medium. Very few materials exhibit such low elastic parameters, and seeking for resonators made of a material commonly used in nano or microfabrication, whose lateral dimensions are much smaller than the wavelength and that allow controlling the propagation of elastic waves in solids is therefore of primary importance for designing new acoustical metamaterials.

Actually, these properties can be found in some phononic crystals that may exhibit local resonances at frequencies below the Bragg band gap, giving rise to one or several forbidden bands in a frequency range where the wavelength is much larger than the period. This has been demonstrated both numerically and experimentally, with one-dimensional stripes periodically engraved on the surface of a lithium niobate substrate [8] and more recently with two-dimensional phononic crystals made of a periodical array of cylindrical pillars deposited on a thin and homogeneous slab [9-18]. This last structure deserves special attention. Indeed, a pillar exhibits both compressional (monopolar) and bending (dipolar) resonances that may lead to negative dynamic effective modulus and mass density, respectively [3]. Moreover, the resonant frequencies can be easily tuned through a proper choice of the height and/or the diameter of the pillar. Finally, since the compressional resonant frequency (monopolar) and the bending resonant frequency (dipolar) are mainly sensitive to the height and to the diameter, respectively, they can be tuned almost independently from each other. This allows, in turn, easy probing of the conditions for either the effective 
mass density negativity or the effective modulus negativity to appear.

Given their potential to serve as local resonators in an all-solid elastic metamaterial [19-21], we have investigated in this work their dynamic properties. We have successively considered a single pillar and a line of identical pillars on a thin plate. In both cases, the resonators as well as the plate were made of silicon. We first numerically investigated the normal modes and their dependence on the geometry and dimensions. We then studied numerically their interaction with an antisymmetric Lamb wave propagating in the plate when the frequency is tuned to a resonant frequency of the pillar. We more specifically point to how both the amplitude and the phase of the wave are affected by the scattering on the pillar, or on the line of pillars, when the frequency is tuned on either a compressional or a bending eigenmode. We show, in particular, that for either of both modes, the pillars behave as secondary sources that reemit in the plate a wave $180^{\circ}$ out-of-phase with the incident wave. For specific dimensions of the pillars, both compressional and bending resonances arise at the same frequency, giving rise to peculiar behavior. We describe and analyze these behaviors with special attention paid to the phase of the reemitted waves. We investigate the conditions in which a more or less pronounced minimum of the transmission through the line of pillars results from the superposition of both incident and reemitted waves.

\section{NORMAL MODES OF A SINGLE PILLAR AND OF A LINE OF PILLARS ON A SLAB}

The goal of this section is to provide in a comprehensive way a numerical study of the vibrational states of a single pillar, or a line of identical pillars, erected on a membrane of thickness $t$. We have considered the effect of the usual geometrical parameters, namely, the height $h$, the diameter $d$ of the pillar, and the thickness $t$ of the membrane. It will be shown below that, in the quest for resonators with lateral dimensions much smaller than the wavelength, dealing with pillars featuring an inverted conical shape may be advantageous. We therefore have also considered this case, the half top angle being denoted $\theta$. All calculations have been performed with the help of a finite element method (COMSOL MULTIPHYSICS). It should be noted here that this structure differs from a beam clamped in a wall since the displacements field on the foot is not null (see below). As a consequence, an analytical derivation of the vibrational states of the pillar on the plate is very challenging. In the numerical model depicted Fig. 1, we have considered a pillar in the middle of a circular membrane surrounded by a perfect matched layer (PML) to avoid any reflection from the boundary of the plate. The method we used to compute the resonances is as follows: An oscillating force is applied normally to the surface of the plate, along a line [AB] parallel to the crystallographic direction $\langle 100\rangle$ of the silicon ( $x$ axis in the following) and the resulting displacements are monitored against the frequency on five different points on top of the pillar. We have only investigated the propagation of the antisymmetric Lamb mode $A_{0}$, which features a large out-of-plane component $u_{z}$. This may constitute an advantage in the view of the forthcoming experimental verification of the main results described in this article. Moreover, we have

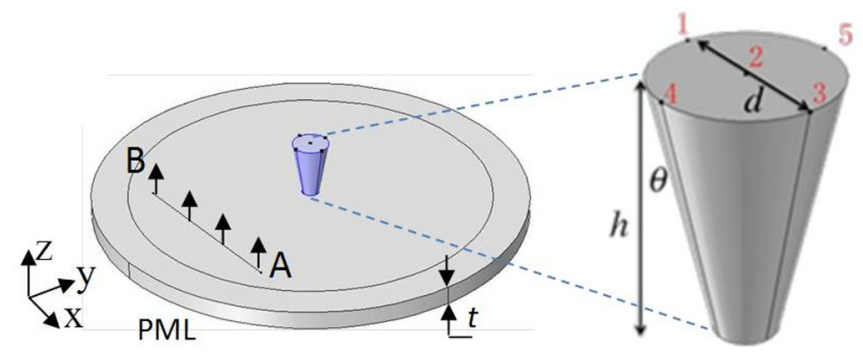

FIG. 1. Model used for finite element calculations. A vertical harmonic force is applied along $[\mathrm{AB}]$. PML is set in the ring surrounding the plate.

only considered $u_{z}$ since no conversion is observed after the interaction of the incident wave with the structure [22,23], i.e., the ratio $u_{z} / u_{x}$ is conserved.

We first have computed $u_{z}$ against the frequency for an isolated cylindrical pillar $\left(\theta=0^{\circ}\right)$ with the parameters $h=245 \mu \mathrm{m}, d=50 \mu \mathrm{m}$, and $t=145 \mu \mathrm{m}$. Each resonance manifests itself by a more or less sharp peak which can be unambiguously ascribed to an eigenmode with the help of the associated displacements [Fig. 2(a)]. Actually, the normal displacements associated to a compressional mode are uniform on the top of the pillar and their amplitude does not depend on points $1-5$ (noted hereafter as $\mathrm{P}_{1-5}$ ) where the measurement is made. On the other hand, a bending mode along the $y$ axis is characterized by out-of-phase displacements in $\mathrm{P}_{4}$ and $\mathrm{P}_{5}$ and no displacement along the line joining $\mathrm{P}_{1}$ and $\mathrm{P}_{3}$, which is a node [Fig. 2(b)]. Therefore, any nonzero displacement measured in $\mathrm{P}_{1}, \mathrm{P}_{2}$, or $\mathrm{P}_{3}$ can be unambiguously ascribed to a compressional mode. The sum and the difference of the normal displacements measured in points 4 and 5 necessarily relate to the compressional and to the bending modes, respectively. We show in Fig. 2(a) both quantities $u_{z}(4)-u_{z}(5)$
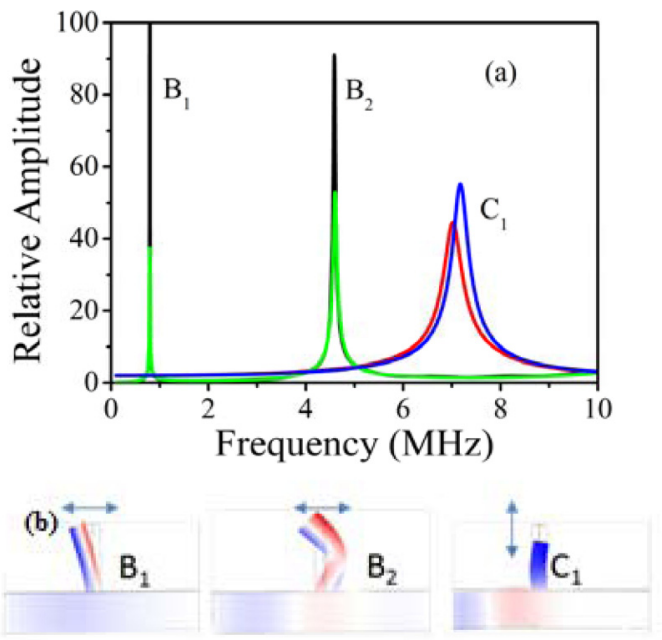

FIG. 2. (a) Resonance frequencies of a single pillar (black and red lines) or a line of pillars (green and blue lines). The dimensions are (in $\mu \mathrm{m}$ ) $h=245, d=50, t=145, \theta=0^{\circ}$. (b) Cartography of the displacement fields at resonance. The red (blue) color corresponds to a positive (negative) displacement with a zero represented with the white color. The deformations have been magnified for better observation. 

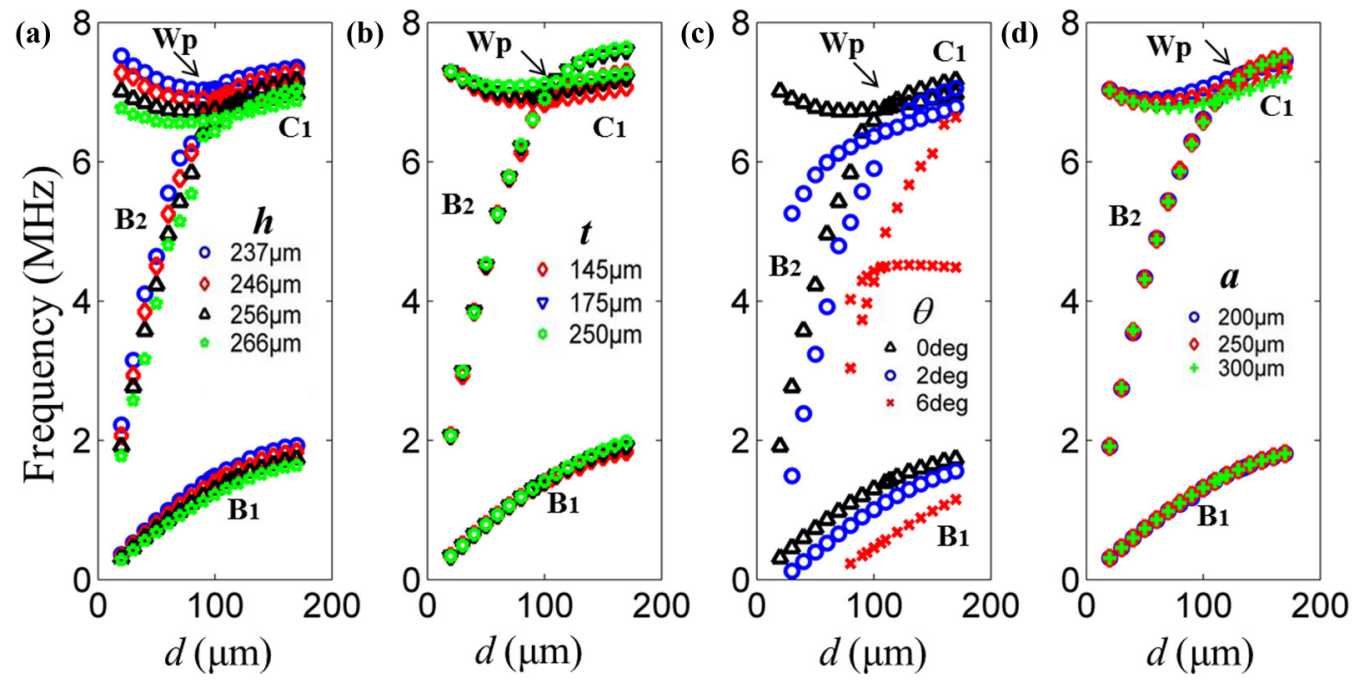

FIG. 3. Variations of the three eigenmodes of a pillar on a plate (simulations) against the diameter $d$ and (a) height $h\left(t=145 \mu \mathrm{m}, \theta=0^{\circ}\right)$, (b) thickness of the plate $t\left(h=246 \mu \mathrm{m}, \theta=0^{\circ}\right)$, (c) half top angle $\theta$ for conical shaped pillar $(h=256 \mu \mathrm{m}, t=145 \mathrm{~mm})$, and (d) lattice constant $a$ when the pillars are regularly spaced in a line $\left(h=256 \mu \mathrm{m}, t=145 \mathrm{~mm}, \theta=0^{\circ}\right)$.

and $u_{z}(4)+u_{z}(5)$ against frequency as a black line and red line, respectively, from which two bending modes (referred to hereafter as $B_{1}$ and $B_{2}$, respectively) at 1.1 and $4.7 \mathrm{MHz}$ and one compressional mode (referred to as $\mathrm{C}_{1}$ ) at $7 \mathrm{MHz}$ can be identified within the explored frequency range $(0-10 \mathrm{MHz})$. This is further confirmed by the displacement fields computed at a moment when the amplitudes are maximum, displayed in Fig. 2(b). All these three resonant frequencies remain relatively unaffected when considering a line of identical pillars separated $200 \mu \mathrm{m}$ from each other. Actually, $\mathrm{B}_{1}$ and $\mathrm{B}_{2}$ still occur on the same frequencies as previously, although with altered relative amplitudes [green line in Fig. 2(a)], whereas $\mathrm{C}_{1}$ now appears at a slightly higher frequency [blue line in Fig. 2(a)] because of a weak coupling through the plate. For each of these modes, the peak width at half maximum is the same for a single pillar and for a line of pillars.

Being due to mechanical resonances, the frequencies and amplitudes of these modes are, though to varying degrees, dependent on the geometrical parameters of the pillar [Figs. 3(a)-3(d)]. We have calculated the resonance frequencies against the diameter for different values of the height [Fig. 3(a)] and the thickness of the supporting plate [Fig. 3(b)]. We have also considered the influences of $\theta$ for a cone-shaped pillar [Fig. 3(c)] and of the distance between pillars when they are arranged in a line [Fig. 3(d)]. One method to obtain the resonance frequencies consists in calculating the rate of elastic energy localized in the pillar through the quantity $\alpha$ :

$$
\alpha=\frac{\iiint_{\text {pillar }} \sqrt{u_{x}^{2}+u_{y}^{2}+u_{z}^{2}} d V}{\iiint_{\text {unit cell }} \sqrt{u_{x}^{2}+u_{y}^{2}+u_{z}^{2}} d V} .
$$

The curves in Fig. 3 were derived from eigenfrequency simulations. We considered a single pillar erected in the center of a $1000 \times 1000 \mu \mathrm{m}^{2}$ plate. Periodic conditions were applied to the four sides of the plate, so that the plate is large enough to reproduce the case of an isolated pillar. The results are in excellent agreement with the resonant frequencies derived from the frequency domain simulations shown in Fig. 2.

Several striking features result from this study. First, one should notice that regardless of the mode, compression or bending, the eigenfrequency decreases for increasing height [Fig. 3(a)]. Then one can tune, almost independently, the bending and the compression resonances through a proper choice of the geometrical parameters. Actually, both the bending modes strongly depend on the diameter $d$ but are less sensitive to the height of the pillar or to the thickness of the plate, in contrast to the compressional mode $\mathrm{C}_{1}$ that can be slightly tuned through the value of $h$ but keeps almost the same frequency upon a change in $d$ or $t$ [Figs. 3(a) and 3(b)]. It should be noted that this eigenmode calculation also allows for outputing a torsional mode that could potentially be excited in the frequency range we have explored. However, as a rotating vibration around the pillar axis, such a mode is characterized by in-plane components $u_{x}$ and $u_{y}$, and the absence of out-of-plane component $u_{z}$. Exciting this mode requires, therefore, exerting a torque parrallel to the plate, which is difficult to achieve within a noncontact experimental scheme. Moreover, the displacements associated with this eigenmode being centrosymmetrical, the coupling with a Lamb wave, either symmetric or antisymmetric, is forbidden.

We have not considered it in this work. We have also investigated how the conicity may influence the eigenmodes. The results for the half top angles $\theta=2^{\circ}$ and $\theta=6^{\circ}$ are displayed in Fig. 3(c).

The comparison with the ideal case of a cylindrical pillar $\left(\theta=0^{\circ}\right)$ demonstrates how sensitive to this parameter the eigenfrequencies are. For the three modes, the conicity makes modes shifting to lower frequencies which, in the context of acoustical metamaterials where the notion of homogenization is essential, is a significant advantage over the perfect cylinder shape.

However, the most salient feature in Figs. 3(a)-3(d) is the crossing of modes $\mathrm{C}_{1}$ and $\mathrm{B}_{2}$ occurring when $d \approx 100 \mu \mathrm{m}$. For this particular geometry, both the monopolar mode $C_{1}$ 

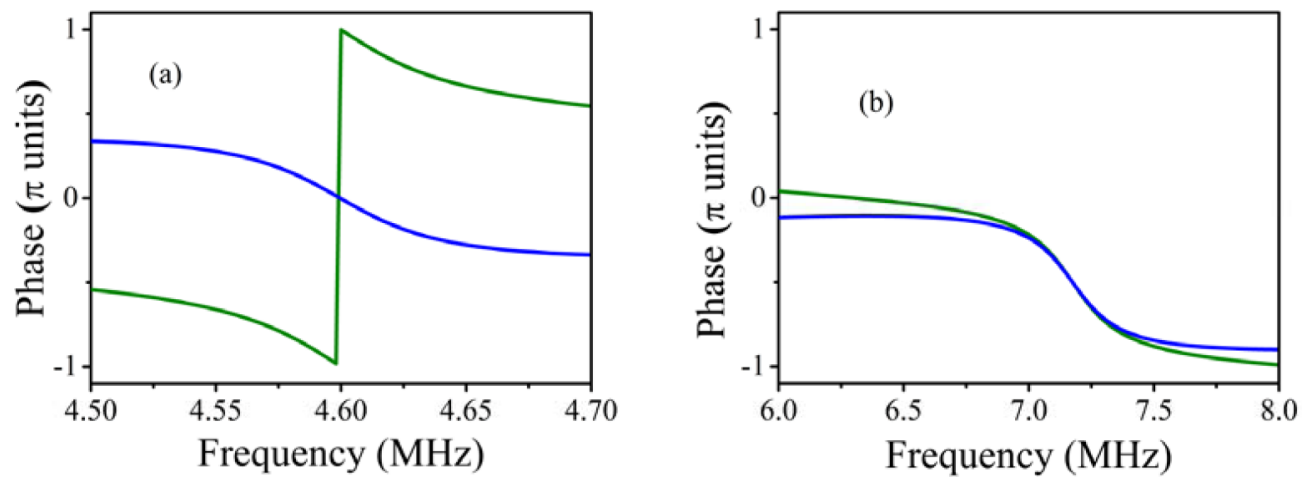

FIG. 4. Phases of the vibrations on top of the pillar measured at $\mathrm{P}_{4}$ (green) and $\mathrm{P}_{5}$ (blue). The reference is the phase on the central axis, at the foot of the pillar. (a) Bending mode $\mathrm{B}_{2}$ and (b) compressional mode $\mathrm{C}_{1}$.

and the dipolar mode $\mathrm{B}_{1}$ coexist, giving rise to a complex motion of the resonator at $\sim 6.5 \mathrm{MHz}$. Much of the importance of investigating both the amplitude and the phase of the displacements around this working point [labeled $\mathrm{W}_{\mathrm{p}}$ in Figs. 3(a)-3(d)] relates to the double-negative behavior with respect to an elastic wave propagating therein, which this class of heterostructures could potentially exhibit.

\section{RELATIVE PHASE BETWEEN THE MOTIONS OF THE PILLAR AND THE SLAB}

\section{A. Single pillar with separated modes}

First we have considered a single pillar with geometric parameters $d=50 \mu \mathrm{m}, t=145 \mu \mathrm{m}, h=245 \mu \mathrm{m}$, and $\theta=$ $0^{\circ}$, for which $\mathrm{C}_{1}$ and $\mathrm{B}_{2}$ arise at distinct frequencies $\left(\nu_{\mathrm{B}_{2}}<\right.$ $v_{\mathrm{C}_{1}}$ ). The variations in phase on both sides of $v_{\mathrm{B}_{2}}=4.6 \mathrm{MHz}$ and $\nu_{\mathrm{C}_{1}}=7 \mathrm{MHz}$, computed at $\mathrm{P}_{4}$ (green curve) and $\mathrm{P}_{5}$ (blue curve), are displayed in Figs. 4(a) and 4(b), respectively. The reference is chosen on the central axis, at the foot of the pillar. Below $\nu_{\mathrm{B}_{2}}$, a phase difference of $-\pi / 2$ (respectively $+^{\pi} / 2$ ) is observed for $\mathrm{P}_{4}$ (respectively $\mathrm{P}_{5}$ ), corresponding for both points to a motion in quadrature between the top and the bottom of the pillar and therefore to an out-of-phase motion between $\mathrm{P}_{4}$ and $\mathrm{P}_{5}$.

The same phase difference of $\pi$ still exists above $\nu_{\mathrm{B}_{2}}$ but with a phase reversal [Fig. 4(a)]. As regards the compressional resonance, all the points on top vibrate in phase with the incident wave for frequencies either lower or larger than $v_{C_{1}}$. For both eigenmodes, a phase shift about $\pi$ occurs when passing through the resonance. Since the wave in the plate can couple to the pillar and excite it on vibration at the frequencies of the eigenmodes, it should be expected that the pillar can in turn trigger a reemitted wave in the plate when vibrating at resonance. One may also expect that the amplitude of the reemitted wave is large enough to significantly interact with the incident wave in the plate. This can be checked by computing the amplitude of the displacement field around the pillar at resonance. This can be easily done by subtracting the incident wave with an amplitude set to unity, from the total wave. The result is shown in Figs. 5(a) and 5(b) when the frequency of the incident wave is tuned to $v_{\mathrm{C}_{1}}=7 \mathrm{MHz}$ and $v_{\mathrm{B}_{2}}=4.6 \mathrm{MHz}$, respectively.

In both cases, the amplitude distribution features the symmetry of the mode, monopolar for $\mathrm{C}_{1}$ and dipolar for
$\mathrm{B}_{2}$, thereby supporting that the pillar acts as a point source reemitting antisymmetric Lamb waves upon excitation on either of its eigenmodes. Note that a similar behavior was found at $\nu_{\mathrm{B}_{1}}=0.8 \mathrm{MHz}$ (not shown here). The amplitude of the emitted waves against the distance to the pillar is displayed in Fig. 5(c): the amplitude decreases as it goes away from the pillar. For either mode, further multiplying the normalized amplitude of the emitted waves by the square root of the distance to the pillar leads to a quantity close to a constant, matching the energy law of a point source and unambiguously establishing that the pillar behaves as an elastic source at resonance [Fig. 5(d)].
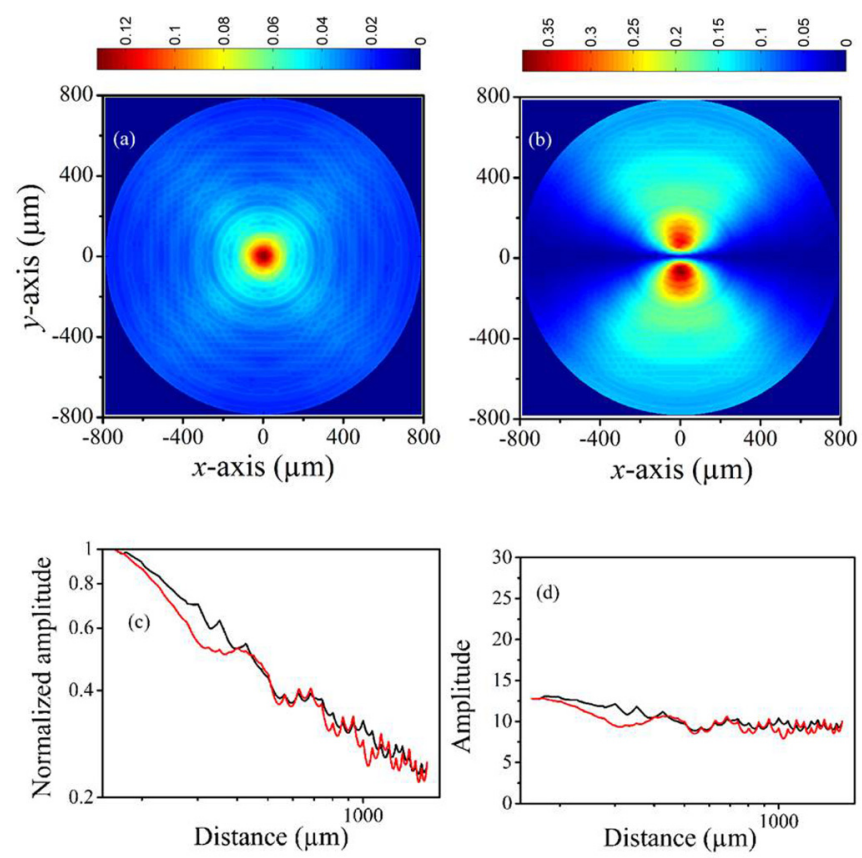

FIG. 5. Upper panels: amplitude distribution of the Lamb waves emitted by the pillar at resonance (a) $\mathrm{C}_{1}$ and (b) $\mathrm{B}_{2}$ modes. Lower panels: (c) normalized displacement amplitudes of the emitted wave against the distance to the pillar, and (d) reported quantity as a result of multiplying the normalized amplitude by the square root of the distance to the pillar. The red line and the black line are for $\mathrm{C}_{1}$ and $\mathrm{B}_{2}$, respectively. 


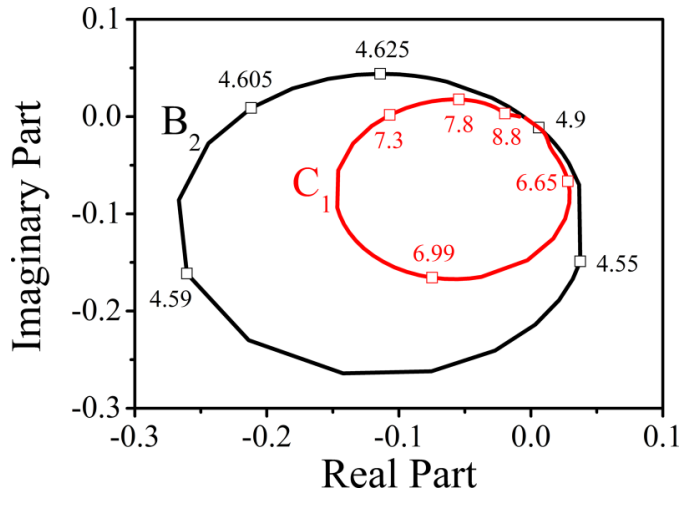

FIG. 6. Parametric plot of the Lamb waves emitted by a single pillar for frequencies around the bending mode $\mathrm{B}_{2}$ (black curve) and the compressional mode $\mathrm{C}_{1}$ (red curve). The numbers are for specific values of the frequency (in $\mathrm{MHz}$ ).

As a consequence of the preceding analysis, the displacement field in the plate at resonance can be regarded as the sum of the incident and emitted waves and therefore, subtracting the former from the total wave allows us to thoroughly analyze the complex amplitude of the latter. We have computed that way the real and imaginary parts of the waves reemitted by a single pillar $\left(d=50 \mu \mathrm{m}, t=145 \mu \mathrm{m}, h=245 \mu \mathrm{m}, \theta=0^{\circ}\right)$ when the frequency is in the vicinity of $\nu_{\mathrm{B}_{2}}=4.6 \mathrm{MHz}$ or $v_{\mathrm{C}_{1}}=7 \mathrm{MHz}$. The calculation is made on a point on the normal to the wave front passing through the center of the pillar and being distant $500 \mu \mathrm{m}$ from it. The amplitude is normalized to that of the incident wave. The results, displayed as a Nyquist plot, are shown in Fig. 6. We have also plotted in this figure the complex value of the emitted wave for some specific frequencies in the explored ranges.

For both resonances, the complex amplitude against frequency takes the form of an ellipse that passes through the origin (no emitted waves at the corresponding frequencies) and that crosses the real line at $v_{\mathrm{B}_{2}}=4.605 \mathrm{MHz}$ and $v_{\mathrm{C}_{1}}=$ 7.3 MHz. At both these frequencies, the real part of the emitted waves is negative and hence the phase with respect to the incident wave is $\pi$ for both. However, the amplitudes are much smaller than the incident wave $\left(0.21\right.$ and 0.1 for $B_{2}$ and $\mathrm{C}_{1}$, respectively) and the motion at the measuring point is essentially that of the incident wave.

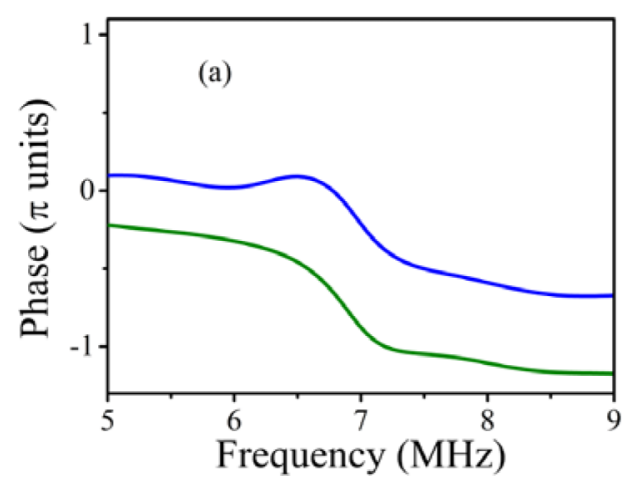

\section{B. Single pillar with superposed modes}

When the dimensions of the pillar are such that the resonances $\mathrm{C}_{1}$ and $\mathrm{B}_{2}$ arise at the same frequency, it can be anticipated that the phase relationships between $\mathrm{P}_{4}$ and $\mathrm{P}_{5}$ are modified due to the combination of the bending and compression motions. This is actually what is shown by the numerical simulations in Fig. 7(a), performed considering a resonator with dimensions $d=100 \mu \mathrm{m}, h=246 \mu \mathrm{m}$, and $t=$ $145 \mu \mathrm{m}$. The phase difference between the vibrations in $\mathrm{P}_{4}$ and $P_{5}$ keeps a constant value for frequencies above the resonance occurring at $v_{\mathrm{C}_{1} / \mathrm{B}_{2}}=6.9 \mathrm{MHz}$ for this geometry [see Figs. 3(a) and 3(b) (red diamonds), and 3(c) (black triangles)]. Let us note that $\mathrm{P}_{4}$ and $\mathrm{P}_{5}$ cannot vibrate in phase because of the bending motion. More interesting are the amplitude and the phase of the emitted waves when the frequency is tuned to $v_{\mathrm{C}_{1} / \mathrm{B}_{2}}$. Actually, as previously, the single pillar acts then as an elastic source that generates an antisymmetric Lamb wave $\pi$ shifted with respect to the exciting wave when the frequency is tuned to $7.55 \mathrm{MHz}$, a value close to the resonance frequency but not equal to it. A relative amplitude of 0.22 is then observed, essentially the same as the amplitude generated by $\mathrm{B}_{2}$ but twice as large as that generated by $\mathrm{C}_{1}$ when the two resonances arise separately [Fig. 7(b)].

Such a behavior should clearly have an impact on the transmission through a line of identical pillars, which is expected to differ whether $B_{2}$ and $C_{1}$ occur at the same frequency or not. We handle this issue in the next section.

\section{Transmission through a line of pillars}

Unlike the single pillar that behaves as a point source reemitting circular waves at resonance (see Fig. 5), it can be anticipated that, thanks to Huygens-Fresnel principle, plane waves ensue from the excitation of identical pillars regularly spaced along an infinite line. According to this principle [24], the infinite adding of single emitted wave along $x$ direction with repeat distance $a$ will produce a result similar to one real line of pillars. In Fig. 8(a) are displayed the phase and the amplitude distribution of the wave emitted by a single pillar at $\mathrm{C}_{1}$ frequency, corresponding to Fig. 5(a). In Fig. 8(b), five identical emitting sources are added with an interdistance $a=200 \mu \mathrm{m}$, which makes the total emitted wave front more flat in the middle. The central five dots stand for the positions of pillars. The same operation is taken for

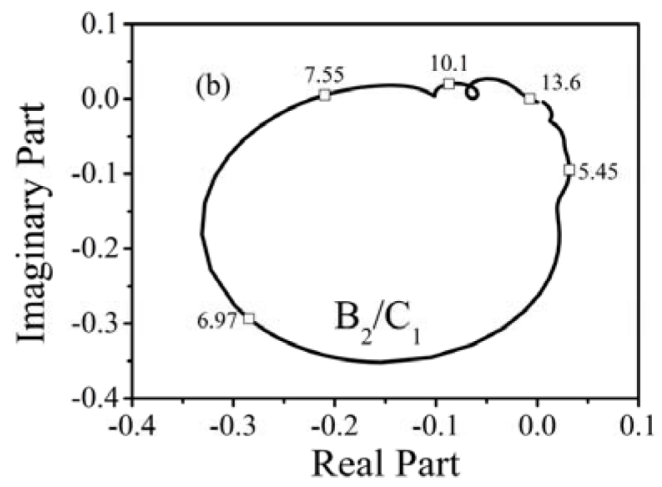

FIG. 7. (a) Phases of the vibrations on top of the single pillar measured at $\mathrm{P}_{4}$ (green) and $\mathrm{P}_{5}$ (blue) when $\mathrm{B}_{2}$ and $\mathrm{C}_{1}$ arise at the same frequency. (b) Parametric plot of the Lamb wave emitted by the pillar around the resonance frequency. 

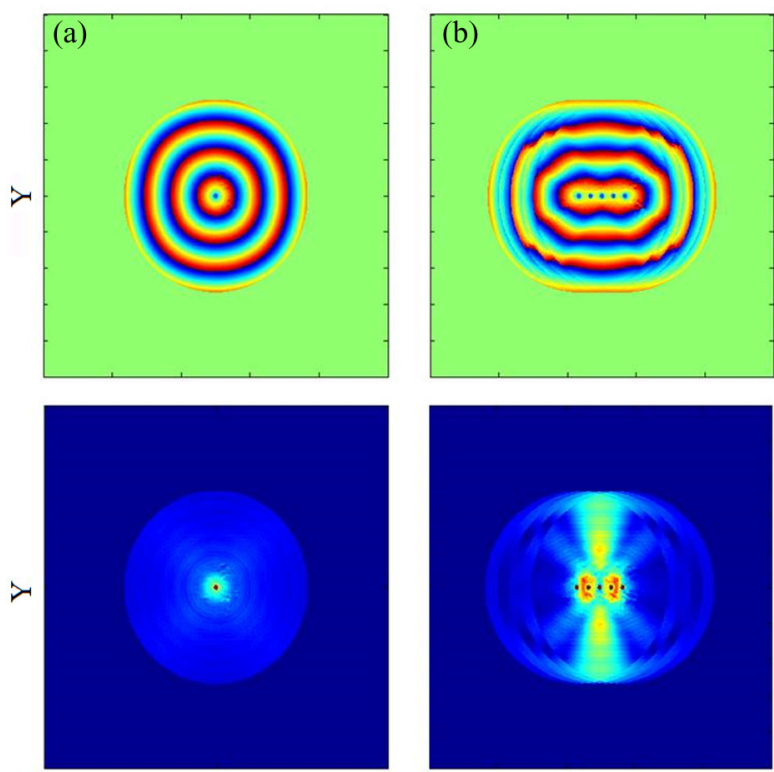

$\mathrm{X}$

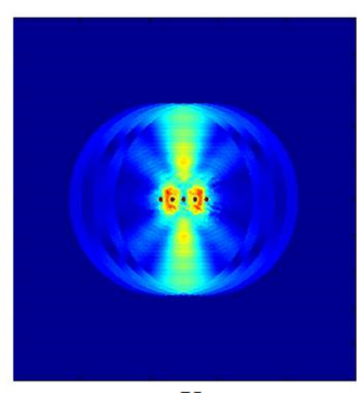

$\mathrm{X}$
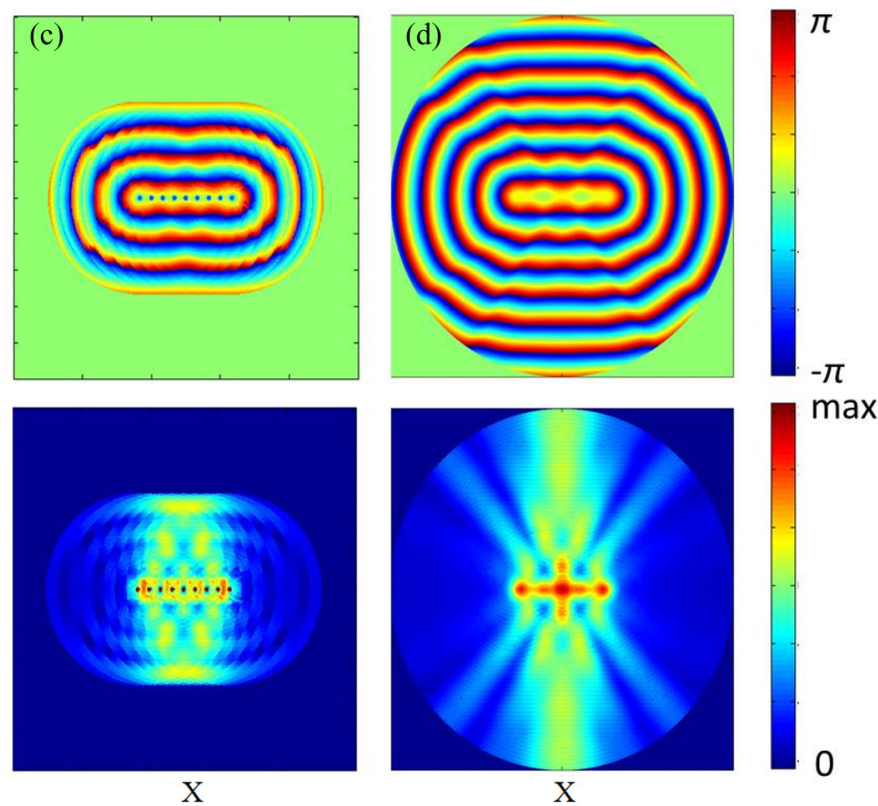

FIG. 8. Phase (upper panel) and amplitude (lower panel) of (a) emitted wave at the frequency $\mathrm{C}_{1}$; (b) adding five single emitting sources at $\mathrm{C}_{1}$ frequency, with the interdistance as lattice parameter $a=200 \mu \mathrm{m}$; (c) similar as in (b) but adding nine single emitting sources at $\mathrm{C}_{1}$ frequency; and (d) emitted wave by one real line of nine pillars with inter distance $a=200 \mu \mathrm{m}$.

adding nine single emitting sources with results in Fig. 8(c), from which we can see the total emitted wave front in $y$ direction is close to a plane wave. Figure 8(d) presents the emitted wave by a real line of nine pillars with interdistance being $a=200 \mu \mathrm{m}$. The sizes of squares for all subgraphs in Fig. 8 are the same, $6000 \times 6000 \mu \mathrm{m}^{2}$. The procedure in this figure supports the Huygens-Fresnel principle: one infinite line of pillars at resonant frequencies will emit plane waves.

The choice of the periodicity $a=200 \mu \mathrm{m}$ results from a compromise. On the one hand, the periodicity should be larger than $\sim 150 \mu \mathrm{m}$ to avoid possible coupling between the compressional motions of neighboring pillars. On the other hand, although the Huygens-Fresnel principle does not impose any upper limit, the periodicity should not be larger than $\sim 300 \mu \mathrm{m}$ in order to reduce the whole size of the structure and avoid very heavy numerical calculations. We also have checked that a pillar isolated on the plate or being an

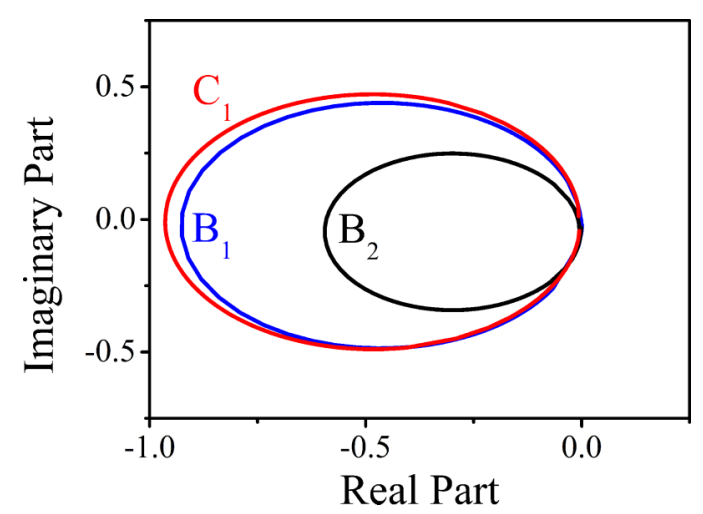

FIG. 9. Nyquist plot of the Lamb waves emitted by a pillar in a line for frequencies around the main three resonances. element of a line behaves the same way at resonance. We made the calculation for pillars with dimensions as above ( $d=50 \mu \mathrm{m}, t=145 \mu \mathrm{m}, h=245 \mu \mathrm{m})$ and for the distance between two adjacent pillars equal to $a=200 \mu \mathrm{m}$. Whatever the normal mode one considers, the resonance frequencies $\left(v_{\mathrm{B}_{1}}=0.8 \mathrm{MHz}, v_{\mathrm{B}_{2}}=4.6 \mathrm{MHz}, v_{\mathrm{C}_{1}}=7.2 \mathrm{MHz}\right)$ and the phase on top of the pillar remain almost unchanged for the pillars in the line [Fig. 2(a)]. Moreover, for the three resonances, the imaginary part of the reemitted waves has a value of zero (Fig. 9) and therefore they are out-of-phase with respect to the incident waves. As regards their relative amplitude, it is about 0.6 for $\mathrm{B}_{2}$ and almost equal to 1 for both $\mathrm{B}_{1}$ and $\mathrm{C}_{1}$, and consequently, the spectrum of the transmission through the line of the pillar has a quasinull value at both these latter frequencies, as shown in Fig. 10. Naturally, the amplitude of the reemitted plane waves depends on the geometry, and minima at other frequencies may be obtained through a proper choice of the dimensions.

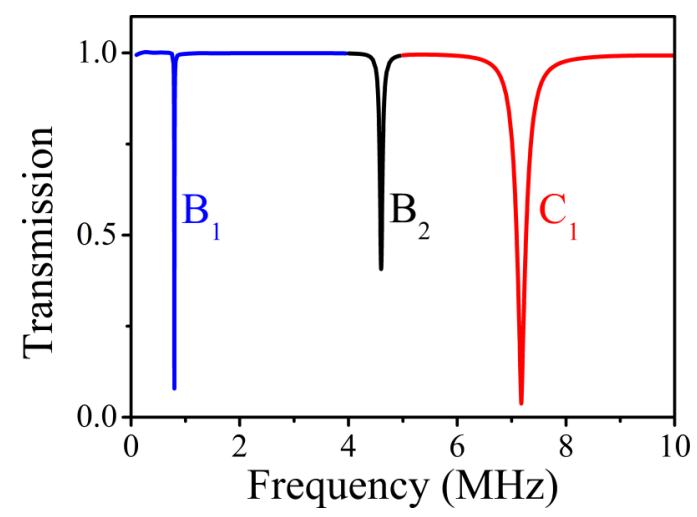

FIG. 10. Transmission through a line of pillars for which $\mathrm{C}_{1}$ (red) and $\mathrm{B}_{2}$ (black) arise at different frequencies. 


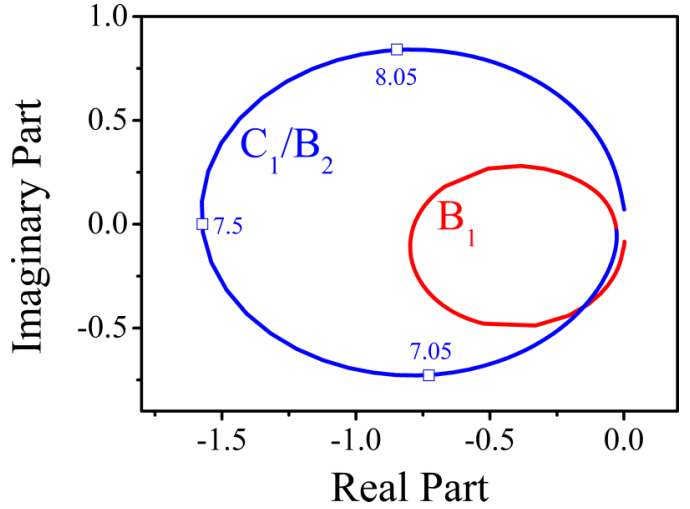

FIG. 11. Nyquist plot of the Lamb waves emitted by a pillar when $\mathrm{C}_{1}$ and $\mathrm{B}_{2}$ arise at the same frequency.

The question which obviously arises now is how these results are modified when both $\mathrm{C}_{1}$ and $\mathrm{B}_{2}$ occur at the same frequency? In fact, our numerical simulations show that, other geometrical parameters being equal, the diameter of the pillars inserted in a line must be slightly larger than that of the single pillar. In that case the common resonance frequency is $v_{\mathrm{C}_{1} / \mathrm{B}_{2}} \approx 7.5 \mathrm{MHz}$ when the diameter is $d=112 \mu \mathrm{m}$ instead of $\nu_{\mathrm{C}_{1} / \mathrm{B}_{2}} \approx 7 \mathrm{MHz}$ and $d=100 \mu \mathrm{m}$ for the isolated pillar. Concerning the phase at points $\mathrm{P}_{4}$ and $\mathrm{P}_{5}$ on top of the resonators, the same behavior as that displayed in Fig. 7(a) for a single pillar is observed. Actually, the most prominent difference lies in the amplitude of the reemitted waves at resonance. We show in Fig. 11 the Nyquist plot of the reemitted waves for frequencies on both sides of $\nu_{\mathrm{B}_{1}}$ (red curve) and $v_{\mathrm{C}_{1} / \mathrm{B}_{2}}$ (blue curve). As previously, the real part is negative and the imaginary part becomes null at resonance for both, meaning that the waves are emitted with a $\pi$ shift with respect to the incident wave. Note also the quasisymmetry with respect to the real part axis of the plots in Fig. 11 and the $\pm^{3 \pi} / 4$ phase shift at the frequencies on the minor axis of the ellipse, namely 7.05 and $8.05 \mathrm{MHz}$. However, whereas no change is observed for the amplitude of $B_{1}(0.79$ whether or not the pillar is isolated $)$, the amplitude of $\mathrm{C}_{1} / \mathrm{B}_{2}$ gets significantly higher than both the amplitudes observed at resonance when the two modes arise at different frequencies. The amplitude at $v_{\mathrm{C}_{1} / \mathrm{B}_{2}}$ is now 1.57 , to be compared with 1 at $v_{\mathrm{C}_{1}}$ and 0.59 at $v_{\mathrm{B}_{2}}$ for a line of pillars featuring distinct frequencies for modes $B_{2}$ and $C_{1}$.

The most interesting point here is that the amplitude of the reemitted wave at $v_{\mathrm{C}_{1} / \mathrm{B}_{2}}$ gets larger than that of the incident wave. As a consequence, it can be expected that, unlike the former case, the line of pillars is now transparent to the incident elastic waves, at least partially. This can be further checked by computing the transmission coefficient against the frequency for an infinite line. The result shown in Fig. 12(a) (blue line) together with the displacements evaluated on top of the pillars (black and red lines) confirm that partial transmission (0.57) at $v_{\mathrm{C}_{1} / \mathrm{B}_{2}} \approx 7.5 \mathrm{MHz}$ actually occurs. From Fig. 12(b), the phase of the transmitted wave at $v_{\mathrm{C}_{1} / \mathrm{B}_{2}}$ is out-of-phase ( $\pi$ shift) with respect to the incident wave, supporting that this partial transmitted wave is induced by an emitted wave.

\section{CONCLUSION}

In this paper, we have thoroughly analyzed the Lamb waves scattered by a pillar or a line of pillars at resonance, paying particular attention to the phase. We have shown that the wave in the far field can be considered as the sum of the incident wave and a wave emitted by the resonator or by the line of resonators. The amplitude of this wave decreases as $D^{-1 / 2}$, where $D$ is the distance from the pillar. Far from the resonance, no phase shift is observed, since the pillars simply follow the up and down motion of the surface and the transmission is equal to 1 . At resonance or close to it, the transmission spectrum is different depending on whether the bending and the compression occur at the same frequency or not. When these two modes occur at different frequencies, the total transmitted amplitude is almost null for both. This must be ascribed to the interferences between incident and $\pi$-shifted emitted waves, both having comparable amplitudes. In contrast, the amplitude of the emitted wave gets significantly enhanced when the geometry of the structure is such that both the bending and compression mode occur at the same frequency. In that case, even though there is still a $\pi$ phase shift between incident and emitted waves, transmission of a substantial part of the elastic energy becomes possible. For both the first-order compressional and the second-order bending modes, the wavelength in the plate is much larger than the lateral dimensions of the resonators. On
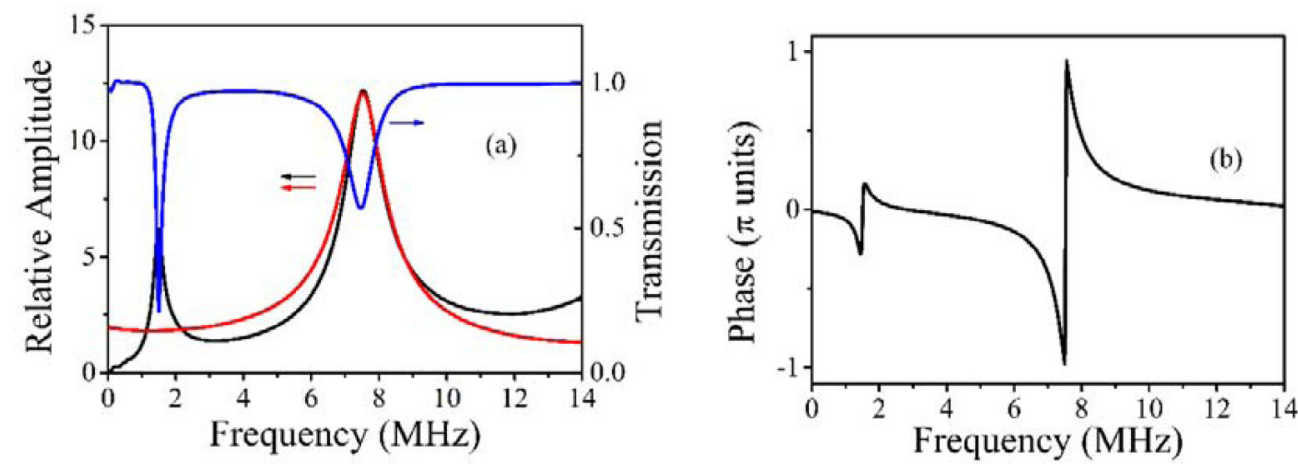

FIG. 12. (a) Amplitudes normalized to the incident wave of the compressional mode $\mathrm{C}_{1}$ (red curve) and bending mode $\mathrm{B}_{2}$ (black curve) with red and black arrows corresponding to the left $y$ axis. Transmission of the antisymmetric Lamb mode through the line of pillars is marked as a blue curve, with a blue arrow corresponding to the right $y$ axis. (b) Normalized phase of transmitted wave with respect to incident wave. 
the other hand, they have a marked monopolar and dipolar character, respectively, and therefore the analysis of this pillar-type metasurface in terms of effective compressibility (related to a monopolar mode) and/or effective mass density (related to a dipolar mode) is relevant.

\section{ACKNOWLEDGMENTS}

This work is supported by the Agence Nationale de la Recherche and the Direction Générale de l'Armement under Grant No. ANR 11 ASTR 015.
[1] G. Ma and P. Sheng, Sci. Adv. 2, e1501595 (2016).

[2] Z. Liu, X. Zhang, Y. Mao, Y. Y. Zhu, Z. Yang, C. T. Chan, and P. Sheng, Science 289, 1734 (2000).

[3] J. Li and C. T. Chan, Phys. Rev. E 70, 055602(R) (2004).

[4] J. Pierre, B. Dollet, and V. Leroy, Phys. Rev. Lett. 112, 148307 (2014).

[5] T. Brunet, J. Leng, and O. Mondain-Monval, Science 342, 323 (2013).

[6] T. Brunet, A. Merlin, B. Mascaro, K. Zimny, L. Leng, O. Poncelet, C. Aristegui, and O. Mondain-Monval, Nat. Mater. 14, 384 (2015).

[7] T. Still, M. Oudich, G. K. Auerhammer, D. Vlassopoulos, B. Djafari-Rouhani, G. Fytas, and P. Sheng, Phys. Rev. B 88, 094102 (2013).

[8] V. Laude, L. Robert, W. Daniau, A. Khelif, and S. Ballandras, Appl. Phys. Lett. 89, 083515 (2006).

[9] Y. Pennec, B. Djafari-Rouhani, H. Larabi, J. O. Vasseur, and A.-C. Hladky-Hennion, Phys. Rev. B 78, 104105 (2008).

[10] Y. Pennec, B. Djafari Rouhani, H. Larabi, A. Akjouj, J. N. Gillet, J. O. Vasseur, and G. Thabet, Phys. Rev. B 80, 144302 (2009).

[11] T. T. Wu, Z. G. Huang, T. C. Tsai, and T. C. Wu, Appl. Phys. Lett. 93, 111902 (2008).

[12] T. C. Wu, T. T. Wu, and J. C. Hsu, Phys. Rev. B 79, 104306 (2009).

[13] B. Djafari-Rouhani, J. O. Vasseur, A. C. Hladky-Hennion, P. Deymier, F. Duval, B. Dubus, and Y. Pennec, Phot. Nano. Fund. Appl. 6, 32 (2008).
[14] R. Pourabolghasem, S. Mohammadi, A. A. Eftekhar, A. Khelif, and A. Adibi, Appl. Phys. Lett. 105, 231908 (2014).

[15] M. Oudich, B. Djafari-Rouhani, Y. Pennec, M. B. Assouar, and B. Bonello, J. Appl. Phys. 116, 184504 (2014).

[16] Y. Jin, N. Fernez, Y. Pennec, B. Bonello, R. P. Moiseyenko, S. Hemon, Y. Pan, and B. Djafari-Rouhani, Phys. Rev. B 93, 054109 (2016).

[17] Y. Jin, Y. Pennec, Y. Pan, and B. Djafari-Rouhani, Crystals 6, 64 (2016).

[18] Y. Jin, Y. Pennec, Y. Pan, and B. Djafari-Rouhani, J. Phys. D: Appl. Phys. 50, 035301 (2017).

[19] A. Colombi, P. Roux, S. Guenneau, and M. Rupin, J. Acoust. Soc. Am. 137, 1783 (2015).

[20] M. Rupin, F. Lemoult, G. Lerosey, and P. Roux, Phys. Rev. Lett. 112, 234301 (2014).

[21] M. Rupin, P. Roux, G. Lerosey, and F. Lemoult, Sci. Rep. 5, 13714 (2015).

[22] Y. Jin, D. Torrent, Y. Pennec, G. Leveque, Y. Pan, and B. Djafari-Rouhani, AIP Adv. 6, 121602 (2016).

[23] Y. Jin, D. Torrent, and B. Djafari-Rouhani, J. Phys. D: Appl. Phys. 50, 225301 (2017).

[24] M. Born, E. Wolf, and A. B. Bhatia, Principles of $O p$ tics: Electromagnetic Theory of Propagation, Interference and Diffraction of Light (Cambridge University Press, Cambridge, UK, 1999). 